\title{
Chaos Synchronization of Uncertain Lorenz System via Single State Variable Feedback
}

\author{
Fengxiang Chen ${ }^{1,2 *}$, Tong Zhang ${ }^{1,2}$ \\ ${ }^{1}$ School of Automotive Studies, Tongji University, Shanghai, China \\ ${ }^{2}$ Clean Energy Automotive Engineering Center, Tongji University, Shanghai, China \\ Email: Fxchen_qjy@hotmail.com
}

Received May 6, 2013; revised June 6, 2013; accepted June 13, 2013

Copyright (c) 2013 Fengxiang Chen, Tong Zhang. This is an open access article distributed under the Creative Commons Attribution License, which permits unrestricted use, distribution, and reproduction in any medium, provided the original work is properly cited.

\begin{abstract}
This paper treats the problem of chaos synchronization for uncertain Lorenz system via single state variable information of the master system. By the Lyapunov stability theory and adaptive technique, the derived controller is featured as follows: 1) only single state variable information of the master system is needed; 2) chaos synchronization can also be achieved even if the perturbation occurs in some parameters of the master chaotic system. Finally, the effectiveness of the proposed controllers is also illustrated by the simulations as well as rigorous mathematical proofs.
\end{abstract}

Keywords: Uncertain Lorenz System; Single State Variable; Chaos Synchronization

\section{Introduction}

Chaos control and synchronization have been intensively investigated during last decade [1-3] and still have attracted increasing attention in recent years. Chaos synchronization has many potential applications in secure communication [1], laser physics, chemical reactor process [2], biomedicine and so on. Up to now, numerous methods have been proposed to cope with the chaos synchronization, such as backstepping design method [3], adaptive design method [4], impulsive control method [5], sliding mode control method [6,7], and other control methods [8-10]. But most of the proposed methods abovementioned need more single state variable information of the master system. However, for instance, the more state variables transmitted to the slave system means the more bandwidth and energy consumption in secure communication system as well as security reduction. Additionally, controller based on single state variable is simple, efficient and easy to be implemented in practical applications [11]. For example, in a real engineering case, some state variables may be difficult or even cannot be detected.

Recently, scholars begin to have attention on the problem of chaos synchronization via single state variable controller (hereafter refereed to as "SSVC") with the motivation of the above facts. Jiang Zhang [12] gives a

\footnotetext{
*Corresponding author.
}

schematic method to design the synchronization controller for a class of chaos system based on backstepping design, and several elegant results derived. However, the controllers conceived by several high-degree complex polynomials. M. T. Yassen [11] provided linear SSVC to Lu chaotic system, but the gain of the controller is difficult to be determined due to the fact that it contains the information of the upper bound of the system trajectory. In order to overcome the deficiency, he modified the SSVC based on adaptive technique. Junan Lu [13] gave out an adaptive SSVC for an unified chaotic system (unification of Lorenz, Chen and Lu chaotic system). Fengxiang Chen [14] proposes a linear SSVC for Lu system via theory of cascade-connection system. However, literatures [11-14] did not take the parameters uncertainty into account, and the synchronization failed when some uncertainty occurs. On the other hand, for an electrical or electronic system, parameter uncertainty is inevitably suffered due to the variation of temperature, humidity, voltage, or interference of electric and magnetic fields. Thus, in this paper, we will provide robust SSVC for uncertain Lorenz chaotic system based on Lyapunov stability theory, and its effectness is validated by both rigorous theoretical analysis and simulations.

The rest of this paper is organized as follows. In the next section, the problem statement on the scheme of master-slave chaos synchronization for uncertain Lorenz system via single state variable information is presented. 
In Section 3, three controllers are provided to the Lorenz system without/with parameters perturbation. In Section 4 , numerical simulations are provided to illustrate the effectiveness of the proposed controllers. Finally, some conclusion remarks are included in Section 5.

\section{Problem Formulation}

The Lorenz system is a system of ordinary differential equations (the Lorenz equations defined by (1)) first studied by Edward Lorenz [15]. It is notable for having chaotic solutions for certain parameter values and initial conditions. In particular, the Lorenz attractor is a set of chaotic solutions of the Lorenz system. Consider the Lorenz system:

$$
\begin{aligned}
& \dot{x}_{1}=\sigma\left(x_{2}-x_{1}\right) \\
& \dot{x}_{2}=r x_{1}-x_{2}-x_{1} x_{3} \\
& \dot{x}_{3}=x_{1} x_{2}-b x_{3}
\end{aligned}
$$

where $\sigma, r, b$ are system parameters, $x_{1}, x_{2}, x_{3}$ are state variables, the system generates the chaotic behavior (see Figure 1) when $\sigma=10, r=28, b=8 / 3$. Hereafter in this paper, we refer to the system (1) as master system and assume that $x_{1}$ is the only available state variable.

The related slave system with control inputs are written as

$$
\begin{aligned}
& \dot{\hat{x}}_{1}=\sigma_{0}\left(\hat{x}_{2}-\hat{x}_{1}\right)+u_{1} \\
& \dot{\hat{x}}_{2}=r_{0} \hat{x}_{1}-\hat{x}_{2}-\hat{x}_{1} \hat{x}_{3}+u_{2} \\
& \dot{\hat{x}}_{3}=\hat{x}_{1} \hat{x}_{2}-b_{0} \hat{x}_{3}+u_{3}
\end{aligned}
$$

where $\sigma_{0}, r_{0}, b_{0}$ are system nominal parameters and $\hat{x}_{1}, \hat{x}_{2}, \hat{x}_{3}$ are state variables, $u_{i}=u_{i}\left(x_{1}, \hat{x}_{1}, \hat{x}_{2}, \hat{x}_{3}\right)$, i.e. $u_{i}: D \subset R^{4} \rightarrow R, i=1,2,3$, and they will be work out later.

Our target is to find out the function $u_{i}, i=1,2,3$ such that the trajectory of the slave system (2) is going to asymptotically approach the master system (1) and finally implement chaos synchronization in the sense that

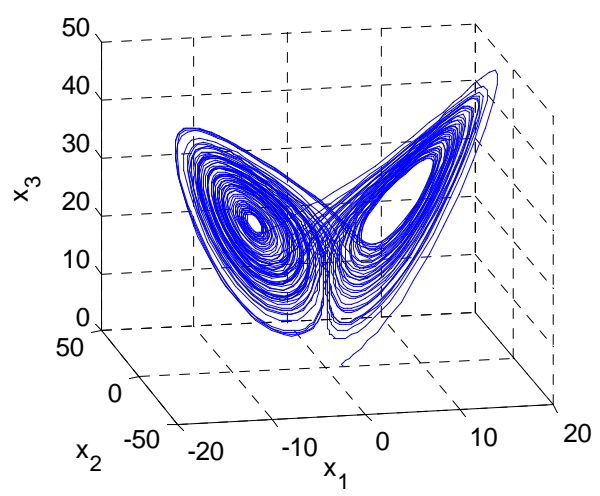

Figure 1. Lorenz chaos phenomenon at $\sigma=10, r=28$, $b=8 / 3$. $\lim _{t \rightarrow \infty} e_{i}=0, i=1,2,3$ where $e_{i}=x_{i}-\hat{x}_{i}$.

\section{Controller Design}

According to the parameter uncertainties, we can classify the synchronization system into 8 cases listed in Table $\mathbf{1}$.

If we introduce the set $A, B, C$ defined as following:

$$
\begin{gathered}
A=\left\{\left(\sigma, r, b, \sigma_{0}, r_{0}, b_{0}\right) \mid \sigma=\sigma_{0}, r=r_{0}, b=b_{0}\right\} \\
B=\left\{\left(\sigma, r, b, \sigma_{0}, r_{0}, b_{0}\right) \mid \sigma \neq \sigma_{0}, r=r_{0}, b=b_{0}\right\} \\
C=\left\{\left(\sigma, r, b, \sigma_{0}, r_{0}, b_{0}\right) \mid\left(r-r_{0}\right)^{2}+\left(b-b_{0}\right)^{2} \neq 0\right\}
\end{gathered}
$$

Obviously, it is an equivalent partition for the element from ${ }_{8}$ case 1 to case 8. i.e., $A=$ case $1, B=$ case 2 , $C=\bigcup$ casei . Consequently, in this section, we are only going ${ }^{-3}$ to investigate the chaos synchronization under three different classifications defined by the set $A, B, C$.

C1: $\left(\sigma, r, b, \sigma_{0}, r_{0}, b_{0}\right) \in A$

Theorem 1: The two Lorenz chaotic systems (1) and (2) can be synchronized under the control law as follows:

$$
\left\{\begin{array}{l}
u_{1}=0 \\
u_{2}=r_{0} x_{1}-r_{0} \hat{x}_{1}+\hat{x}_{1} \hat{x}_{3}-x_{1} \hat{x}_{3} \\
u_{3}=x_{1} \hat{x}_{2}-\hat{x}_{1} \hat{x}_{2}
\end{array}\right.
$$

Proof: Subtracting Equation (1) from Equation (2), then the error dynamic system is obtained as

$$
\begin{aligned}
& \dot{e}_{1}=\sigma_{0}\left(e_{2}-e_{1}\right) \\
& \dot{e}_{2}=-e_{2}-x_{1} e_{3} \\
& \dot{e}_{3}=x_{1} e_{2}-b_{0} e_{3}
\end{aligned}
$$

Choosing the Lyapunov function as

$$
V=\frac{1}{2}\left(\frac{e_{1}^{2}}{\sigma_{0}}+2 e_{2}^{2}+2 e_{3}^{2}\right)
$$

and taking the derivative along the trajectory of the system (4), it yields

$$
\begin{aligned}
\dot{V} & =-e_{1}^{2}+e_{1} e_{2}-2 e_{2}^{2}-2 x_{1} e_{2} e_{3}+2 x_{1} e_{2} e_{3}-2 b_{0} e_{3}^{2} \\
& =-e_{1}^{2}-2 e_{2}^{2}+e_{1} e_{2}-2 b_{0} e_{3}^{2}<0
\end{aligned}
$$

The proof is completed.

C2: $\left(\sigma, r, b, \sigma_{0}, r_{0}, b_{0}\right) \in B$

For this case, the system (1) can be equivalent to the perturbation system as

Table 1. Synchronization system classification based on parameter uncertainties.

\begin{tabular}{llllllll}
\hline Case 1 & Case 2 & Case 3 & Case 4 & Case 5 & Case 6 & Case 7 & Case 8 \\
\hline$\sigma=\sigma_{0}$ & $\sigma \neq \sigma_{0}$ & $\sigma \neq \sigma_{0}$ & $\sigma=\sigma_{0}$ & $\sigma=\sigma_{0}$ & $\sigma \neq \sigma_{0}$ & $\sigma \neq \sigma_{0}$ & $\sigma=\sigma_{0}$ \\
$r=r_{0}$ & $r=r_{0}$ & $r \neq r_{0}$ & $r \neq r_{0}$ & $r \neq r_{0}$ & $r \neq r_{0}$ & $r=r_{0}$ & $r=r_{0}$ \\
$b=b_{0}$ & $b=b_{0}$ & $b \neq b_{0}$ & $b \neq b_{0}$ & $b=b_{0}$ & $b=b_{0}$ & $b \neq b_{0}$ & $b \neq b_{0}$ \\
\hline
\end{tabular}




$$
\begin{aligned}
& \dot{x}_{1}=\sigma_{0}\left(x_{2}-x_{1}\right)+\Delta_{1} \\
& \dot{x}_{2}=r_{0} x_{1}-x_{2}-x_{1} x_{3} \\
& \dot{x}_{3}=x_{1} x_{2}-b_{0} x_{3}
\end{aligned}
$$

where $\Delta_{1}=\left(\sigma-\sigma_{0}\right)\left(x_{2}-x_{1}\right)$.

Since the trajectory of the master system (1) is bounded due to the property of chaos system, $\left|\Delta_{1}\right|$ must be bounded, i.e. $\exists M_{1} \in R^{+}$, s.t. $\left|\Delta_{1}\right| \leq M_{1}$.

Theorem 2: The two Lorenz chaotic systems (1) and (2) can be synchronized under the control law as follows:

$$
\left\{\begin{array}{l}
u_{1}=\theta\left(x_{1}-\hat{x}_{1}\right) /\left|x_{1}-\hat{x}_{1}\right| \\
u_{2}=r_{0} x_{1}-r_{0} \hat{x}_{1}+\hat{x}_{1} \hat{x}_{3}-x_{1} \hat{x}_{3} \\
u_{3}=x_{1} \hat{x}_{2}-\hat{x}_{1} \hat{x}_{2} \\
\dot{\theta}=h\left|x_{1}-\hat{x}_{1}\right|
\end{array}\right.
$$

where $h>0$.

Proof: Subtracting Equation (1) from Equation (2), then the error dynamic system is obtained as

$$
\begin{aligned}
& \dot{e}_{1}=\sigma_{0}\left(e_{2}-e_{1}\right)+\Delta_{1}-\theta e_{1} /\left|e_{1}\right| \\
& \dot{e}_{2}=-e_{2}-x_{1} e_{3} \\
& \dot{e}_{3}=x_{1} e_{2}-b_{0} e_{3}
\end{aligned}
$$

Choosing the Lyapunov function as

$$
V=\frac{1}{2}\left(\frac{e_{1}^{2}}{\sigma_{0}}+2 e_{2}^{2}+2 e_{3}^{2}\right)+\frac{1}{2 \sigma_{0} h}\left(\theta-\theta^{*}\right)^{2}
$$

where $\theta^{*}$ is a constant and will be determined later. Taking the derivative along the trajectory of the system (4), it yields

$$
\begin{aligned}
\dot{V} & =-e_{1}^{2}+e_{1} e_{2}-2 e_{2}^{2}-2 x_{1} e_{2} e_{3}+2 x_{1} e_{2} e_{3}-2 b_{0} e_{3}^{2}+\frac{\Delta_{1} e_{1}}{\sigma_{0}}-\frac{\theta\left|e_{1}\right|}{\sigma_{0}}+\frac{\left(\theta-\theta^{*}\right) \dot{\theta}}{\sigma_{0} h} \\
& \leq-\frac{1}{2} e_{1}^{2}-\frac{3}{2} e_{2}^{2}-2 b_{0} e_{3}^{2}+\frac{\left|\Delta_{1}\right|\left|e_{1}\right|}{\sigma_{0}}-\frac{\theta\left|e_{1}\right|}{\sigma_{0}}+\frac{\left(\theta-\theta^{*}\right)\left|e_{1}\right|}{\sigma_{0}} \\
& =-\frac{1}{2} e_{1}^{2}-\frac{3}{2} e_{2}^{2}-2 b_{0} e_{3}^{2}+\frac{\left|\Delta_{1}\right|\left|e_{1}\right|}{\sigma_{0}}-\frac{\theta^{*}\left|e_{1}\right|}{\sigma_{0}}
\end{aligned}
$$

If we select $\theta^{*} \geq\left|\Delta_{1}\right|$, then $\dot{V}<0$.

Comment 1: Since the controller input component $e_{1} /\left|e_{1}\right|$ (see Equation (8)) is not continuous at $e_{1}=0$, it leads to chattering in the viewpoint of engineering application. In order to overcome this defect, a continuous function $2 \arctan \left(k e_{1}\right) / \pi$ is adopted to substitute the discontinuity function $e_{1} /\left|e_{1}\right|$ based on the conception of variable structure controller design theory, and thus the chattering will be eliminated.

C3: $\left(\sigma, r, b, \sigma_{0}, r_{0}, b_{0}\right) \in C$

In this case, the system (1) can be equivalent to the perturbation system as

$$
\begin{aligned}
& \dot{x}_{1}=\sigma_{0}\left(x_{2}-x_{1}\right)+\Delta_{1} \\
& \dot{x}_{2}=r_{0} x_{1}-x_{2}-x_{1} x_{3}+\Delta_{2} \\
& \dot{x}_{3}=x_{1} x_{2}-b_{0} x_{3}+\Delta_{3}
\end{aligned}
$$

where

$$
\begin{aligned}
& \Delta_{1}=\left(\sigma-\sigma_{0}\right)\left(x_{2}-x_{1}\right) \\
& \Delta_{2}=\left(r-r_{0}\right) x_{1} \\
& \Delta_{2}=\left(b-b_{0}\right) x_{3}
\end{aligned}
$$

Since the trajectory of the master system (1) is bounded, $\left|\Delta_{i}\right|$ must be bounded, i.e. $\exists M_{i} \in R^{+}$, s.t. $\left|\Delta_{i}\right| \leq M_{i}$. Additionally, if $\sigma=\sigma_{0}, r=r_{0}, \quad b=b_{0}$ then $\Delta_{1}=0, \Delta_{2}=0$, and $\Delta_{3}=0$ respectively.
Theorem 3: The two Lorenz chaotic systems (1) and (2) can be synchronized under the control law as follows:

$$
\left\{\begin{array}{l}
u_{1}=\sigma_{0} M /\left(x_{1}-\hat{x}_{1}\right) \\
u_{2}=r_{0} x_{1}-r_{0} \hat{x}_{1}+\hat{x}_{1} \hat{x}_{3}-x_{1} \hat{x}_{3} \\
u_{3}=x_{1} \hat{x}_{2}-\hat{x}_{1} \hat{x}_{2}
\end{array}\right.
$$

where $M=\varepsilon_{1}^{-1}\left(M_{1} /\left(2 \sigma_{0}\right)\right)^{2}+\varepsilon_{2}^{-1} M_{2}^{2}+\varepsilon_{3}^{-1} M_{3}^{2}$, $\varepsilon_{1} \in(0,1 / 2), \varepsilon_{2} \in(0,3 / 2), \varepsilon_{1} \in\left(0,2 b_{0}\right)$.

Proof: Subtracting Equation (1) from Equation (2), then the error dynamic system is obtained as

$$
\begin{aligned}
& \dot{e}_{1}=\sigma_{0}\left(e_{2}-e_{1}\right)+\Delta_{1}-M / e_{1} \\
& \dot{e}_{2}=-e_{2}-x_{1} e_{3}+\Delta_{2} \\
& \dot{e}_{3}=x_{1} e_{2}-b_{0} e_{3}+\Delta_{3}
\end{aligned}
$$

Choosing the Lyapunov function as

$$
V=\frac{1}{2}\left(\frac{e_{1}^{2}}{\sigma_{0}}+2 e_{2}^{2}+2 e_{3}^{2}\right)
$$

and taking the derivative along the trajectory of the system (4), it yields

$$
\begin{aligned}
\dot{V}= & -e_{1}^{2}+e_{1} e_{2}-2 e_{2}^{2}-2 x_{1} e_{2} e_{3}+2 x_{1} e_{2} e_{3}-2 b_{0} e_{3}^{2}-M \\
& +2 \Delta_{1} e_{1} /\left(2 \sigma_{0}\right)+2 \Delta_{2} e_{2}+2 \Delta_{3} e_{3}
\end{aligned}
$$

Note that $2 a b \leq \varepsilon a^{2}+\varepsilon^{-1} b^{2}$, for any $\varepsilon \in R^{+}, a, b \in R$, thus 


$$
\begin{aligned}
\dot{V}= & -e_{1}^{2}-2 e_{2}^{2}+e_{1} e_{2}-2 b_{0} e_{3}^{2}-M \\
& +\varepsilon_{1}^{-1}\left(\Delta_{1} /\left(2 \sigma_{0}\right)\right)^{2}+\varepsilon_{1} e_{1}^{2}+\varepsilon_{2}^{-1} \Delta_{2}^{2}+\varepsilon_{2} e_{2}^{2}+\varepsilon_{3}^{-1} \Delta_{3}^{2}+\varepsilon_{3} e_{3}^{2} \\
= & -\left(1 / 2-\varepsilon_{1}\right) e_{1}^{2}-\left(3 / 2-\varepsilon_{2}\right) e_{2}^{2}-\left(2 b_{0}-\varepsilon_{3}\right) e_{3}^{2}-M \\
& +\varepsilon_{1}^{-1}\left(\Delta_{1} /\left(2 \sigma_{0}\right)\right)^{2}+\varepsilon_{2}^{-1} \Delta_{2}^{2}+\varepsilon_{3}^{-1} \Delta_{3}^{2}
\end{aligned}
$$

Note that $\varepsilon_{1} \in(0,1 / 2), \varepsilon_{2} \in(0,3 / 2), \varepsilon_{1} \in\left(0, b_{0}\right)$, and

$$
M \geq \varepsilon_{1}^{-1}\left(\Delta_{1} /\left(2 \sigma_{0}\right)\right)^{2}+\varepsilon_{2}^{-1} \Delta_{2}^{2}+\varepsilon_{3}^{-1} \Delta_{3}^{2}
$$

then $\dot{V}<0$. The proof is completed.

Comment 2: Although the component of input $u_{1}$ will increase to infinity as $x_{1} \rightarrow \hat{x}_{1}$, so the law is only a conceptional controller and can not be implemented in real application. But this does not mean that the controller law is meaningless. In real implementation, we can modify the $u_{1}$ as

$$
u_{1}= \begin{cases}\sigma_{0} M /\left(x_{1}-\hat{x}_{1}\right) & \left|x_{1}-\hat{x}_{1}\right| \geq r \\ \sigma_{0} M\left(x_{1}-\hat{x}_{1}\right) / r^{2} & \left|x_{1}-\hat{x}_{1}\right|<r\end{cases}
$$

With the modified controller, the synchronization error will not approach to zero, but in the vicinity of the $(0,0,0)$, the details see [16] and simulation 3 . On the other hand, the errors of $e_{2}, e_{3}$ is independent of $e_{1}$ and $u_{1}$, but decided by the following subsystem:

$$
\begin{aligned}
& \dot{e}_{2}=-e_{2}-x_{1} e_{3}+\Delta_{2} \\
& \dot{e}_{3}=x_{1} e_{2}-b_{0} e_{3}+\Delta_{3}
\end{aligned}
$$

which means that any effort on the $u_{1}$ will not have any beneficial to attenuate the fluctuation of $e_{2}, e_{3}$.

\section{Numerical Simulation}

In this section, three numerical simulations named as Simulation 1, Simulation 2 and Simulation 3 are carried out to illustrate the effectiveness of the proposed controller. For sake of simplification, we refer the controller defined by (3) to as nominal controller, the controller defined by (8) to as adaptive controller, and the controller defined by (14) to as robust controller in the following simulations.

\section{Simulation 1:}

In this subsection, we are going to validate the effectiveness of nominal controller designed under the condition $\left(\sigma, r, b, \sigma_{0}, r_{0}, b_{0}\right) \in A$. Thus, three parameters of Lorenz master system and slave system are chosen identically as $\sigma=\sigma_{0}=10, r=r_{0}=28, b=b_{0}=8 / 3$. Initial states of the master and slave system are taken as $x_{1}(0)=2, x_{2}(0)=0, x_{3}(0)=0$ and $\hat{x}_{1}(0)=1.1, \hat{x}_{2}(0)=0.2, \hat{x}_{3}(0)=1$, respectively, and they will be kept the same through out the following simulations. Taking the control input as the nominal controller, the simulation results are shown in Figure 2. As we can
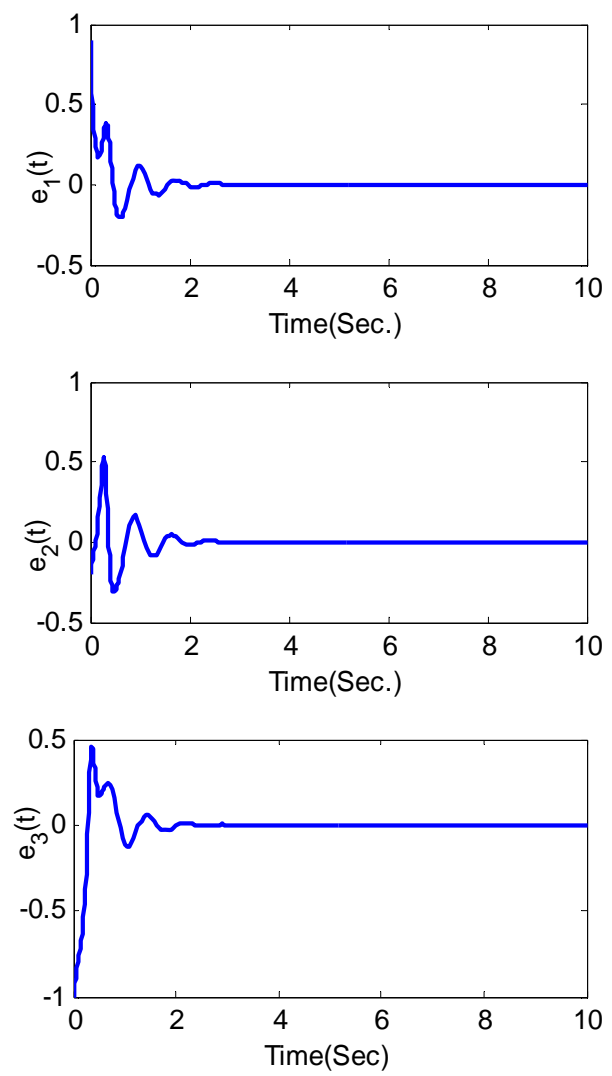

Figure 2. Synchronization errors with the nominal controller for Lorenz system without uncertainty.

see that the synchronization is achieved after about 3 seconds. Which means that the nominal controller is effective for the synchronization system without uncertainty; however, this is not the case when the parameter perturbations are occurred and the results are shown in the subsequently Simulation 2 and Simulation 3.

\section{Simulation 2:}

In this subsection, we add the parameter $30 \%$ perturbations to $\sigma$, i.e., $\sigma=13$, . The adaptive controller defined by (8) is taken as

$$
\left\{\begin{array}{l}
u_{1}=\theta \arctan \left(800\left(x_{1}-\hat{x}_{1}\right)\right) \\
u_{2}=r_{0} x_{1}-r_{0} \hat{x}_{1}+\hat{x}_{1} \hat{x}_{3}-x_{1} \hat{x}_{3} \\
u_{3}=x_{1} \hat{x}_{2}-\hat{x}_{1} \hat{x}_{2} \\
\dot{\theta}=100\left|x_{1}-\hat{x}_{1}\right|, \quad \theta(0)=5
\end{array}\right.
$$

which has been modified according to the Comment 1 . And the results are shown in Figure 3. We can see that the synchronization via nominal controller is failed due to the fluctuation of $e_{1}(t)$. Meanwhile, the synchronization via the adaptive controller is achieved after about 4 seconds, and the adaptive variable $\theta$ goes to a fixed constant. Obviously, the adaptive controller proposed in Theorem 2 really eliminates the disturbance suffered from the parameters perturbation, and the synchronization is 

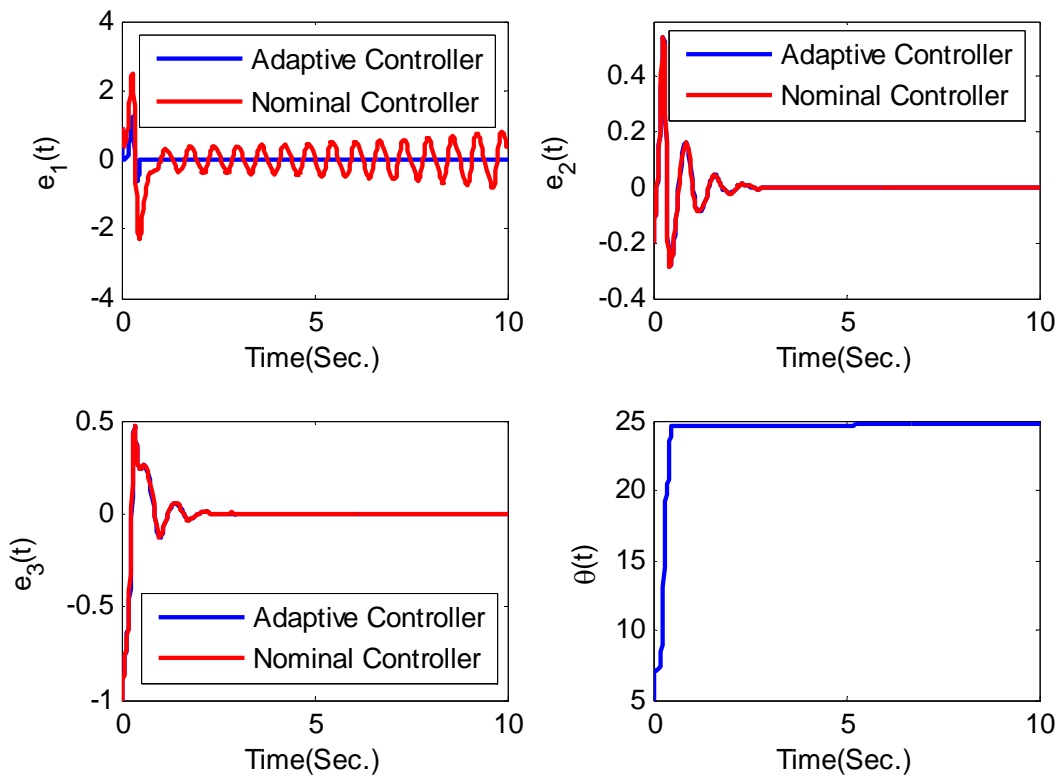

Figure 3. Synchronization errors for uncertain Lorenz system via the adaptive controller and nominal controller, respectively.

achieved. On the other hand, the perturbation of parameter $\sigma=13$ hardly has any impact on the convergence of $e_{2}(t), e_{3}(t)$ due to the subsystem

$$
\begin{aligned}
& \dot{e}_{2}=-e_{2}-x_{1} e_{3} \\
& \dot{e}_{3}=x_{1} e_{2}-b_{0} e_{3}
\end{aligned}
$$

is independent of $\sigma$ and $e_{1}$. It can be easily to be proved that the subsystem (23) is asymptotically stable (i.e. $\hat{x}_{2} \rightarrow x_{2}, \hat{x}_{3} \rightarrow x_{3}$ as $t \rightarrow \infty$ ) with any $x_{1}$. For example, choosing Lyapunov function as $V=e_{2}^{2}+e_{3}^{2}$, then $\dot{V}=-e_{2}^{2}-b_{0} e_{3}^{2}<0$. Which means that the convergence rate of $\hat{x}_{2}, \hat{x}_{3}$ is independent of the perturbation of $\sigma$ and $e_{1}$.

\section{Simulation 3:}

In this subsection, we are going to analysis the performance of robust controller designed under the condition $\left(\sigma, r, b, \sigma_{0}, r_{0}, b_{0}\right) \in C$. Here we consider the worst case, i.e. $\sigma \neq \sigma_{0}, r \neq r_{0}, b \neq b_{0}$. The parameters of the master system are taken as $\sigma=13 \quad(+30 \%$ perturbation of the nominal value 10), $r=23.8 \quad(-15 \%$ perturbation of the nominal value 28$), b=3.2 \quad(+20 \%$ perturbation of the nominal value 8/3). Robust controller and nominal controller are here both adopted so as to compare their performance on the chaos synchronization and the simulation results are shown in Figure 4. From Figure 4, we can see that the synchronization via nominal controller and robust controller are both failed due to the errors do not converge to zero but undulate in a bounded area. For robust controller, $e_{1}(t)$ approaches to zero, but this is not the case for other error component $e_{2}(t)$ and $e_{3}(t)$, which has been interpreted by Comment 2 . For nominal controller, all error components $e_{1}(t), e_{2}(t)$ and
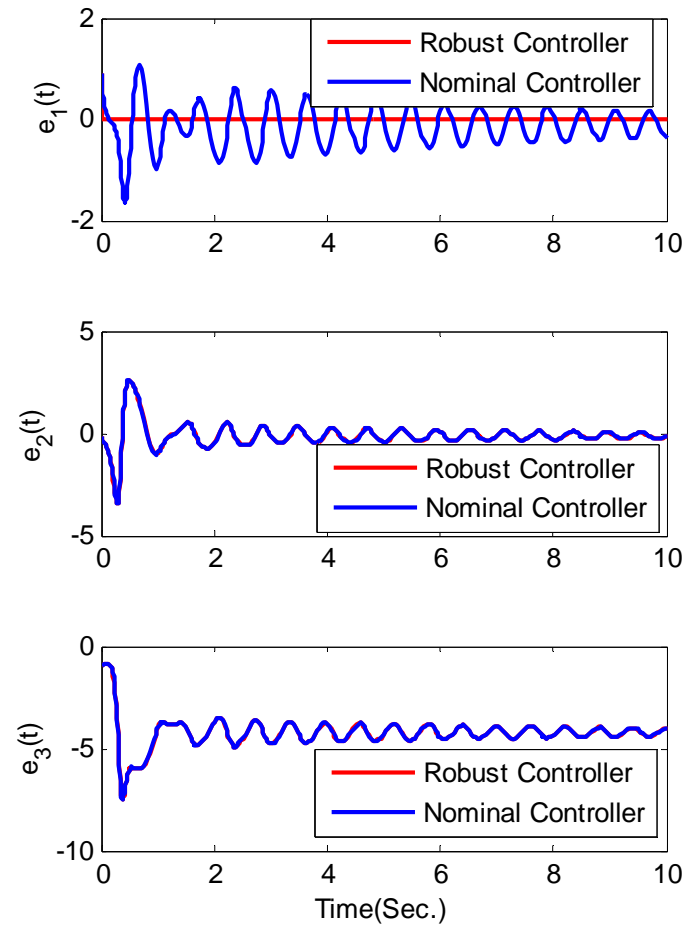

Figure 4. Synchronization errors for uncertain Lorenz system via the robust controller and nominal controller, respectively.

$e_{3}(t)$ varied in the vicinity of certain value, respectively. As mentioned in Comment 2, the error component $e_{2}(t)$ and $e_{3}(t)$ is independent of $e_{1}$ and $u_{1}$, but decided by (21). Which means that both $e_{2}(t)$ and $e_{3}(t)$ must keep the same when different controller (nominal controller and robust controller) acted on the slave system 
and the corresponding numerical simulation result are shown in Figure 4.

\section{Conclusion}

The paper investigates the synchronization of uncertain Lorenz system via feedback of single state variable from the master system. According to parameter uncertainties, an equivalent partition is defined to classify the parameter uncertain Lorenz system. Then three controllers (nominal controller, adaptive controller, and robust controller) are given out to achieve the chaos synchronization based on Lyapunov stability theory and adaptive technique. Finally, three numerical simulations are conducted to validate the effectiveness of the proposed controllers, and it finds that the adaptive controllers do really eliminate the disturbance caused by parameters perturbation but it is not the case for nominal controller. For robust controller, any effort to modify the controller $u_{1}$ will not have beneficial to attenuate the error component $e_{2}(t)$ and $e_{3}(t)$ which is independent of $e_{1}$ and $u_{1}$, but decided by (21). For the future investigation, we will use $L_{2}$ gain theory and passivity theory to design a robust synchronization controller to attenuate the disturbance more effectively based on single state variable.

\section{Acknowledgements}

This work is supported by National Special Fund for the Development of Major Research Equipment and Instruments [2012YQ150256], and National Nature Science Foundation of China [61104077].

\section{REFERENCES}

[1] W. Kinzel, A. Englert and I. Kanter, "On Chaos Synchronization and Secure Communication,” Philosophical Transactions of the Royal Society A, Vol. 368, No. 1911, 2010, pp. 379-389.

http://dx.doi.org/10.1098/rsta.2009.0230

[2] S. Rasoulian and M. Shahrokhi, "Control of a Chemical Reactor with Chaotic Dynamics," Iranian Journal of Chemistry \& Chemical Engineering, Vol. 29, No. 4, 2010, pp. 149-159.

[3] X. Tan, J. Zhang and Y. Yang, "Synchronizing Chaotic Systems Using Backstepping Design,” Chaos, Solitons \& Fractals, Vol. 16, No. 1, 2003, pp. 37-45. http://dx.doi.org/10.1016/S0960-0779(02)00153-4

[4] F. Sorrentino, G. Barlev, A. B. Cohen and E. Ott, "The Stability of Adaptive Synchronization of Chaotic Systems,” Chaos, Vol. 20, No. 1, 2010, Article ID: 013103.

\section{http://dx.doi.org/10.1063/1.3279646}

[5] M. Haeri and M. Dehghani, "Impulsive Synchronization of Different Hyperchaotic (Chaotic) Systems," Chaos, Solitons \& Fractals, Vol. 38, No. 1, 2008, pp. 120-131. http://dx.doi.org/10.1016/j.chaos.2006.10.051

[6] H.-T. Yau, "Design of Adaptive Sliding Mode Controller for Chaos Synchronization with Uncertainties," Chaos, Solitons \& Fractals, Vol. 22, No. 2, 2004, pp. 341-347. http://dx.doi.org/10.1016/j.chaos.2004.02.004

[7] C.-C. Wang and J.-P. Su, “A New Adaptive Variable Structure Control for Chaotic Synchronization and Secure Communication,” Chaos, Solitons \& Fractals, Vol. 20, No. 5, 2004, pp. 967-977. http://dx.doi.org/10.1016/j.chaos.2003.10.026

[8] X. Yu and Y. Song, "Chaos Synchronization via Controlling Partial State of Chaotic Systems,” International Journal Bifurcation and Chaos, Vol. 11, No. 6, 2001, pp. 1737-1741. http://dx.doi.org/10.1142/S0218127401003024

[9] X.-F. Wang, Z.-Q. Wang and G.-R. Chen, “A New Criterion for Synchronization of Coupled Chaotic Oscillators with Application to Chua's Circuits,” International Journal Bifurcation and Chaos, Vol. 9, No. 6, 1999, pp. 11691174. http://dx.doi.org/10.1142/S021812749900081X

[10] J. Cao, H. X. Li and D. W. C. Ho, "Synchronization Criteria of Lur's Systems with Time-Delay Feedback Control," Chaos, Solitons and Fractals, Vol. 23, No. 4, 2005, pp. 1285-1298.

[11] M. T. Yassen, "Feedback and Adaptive Synchronization of Chaotic Lu system,” Chaos Solitons and Fractals, Vol. 25, No. 2, 2005, pp. 379-386. http://dx.doi.org/10.1016/j.chaos.2004.11.042

[12] J. Zhang, C. G. Li, H. B. Zhang and J. B. Yu, "Chaos Synchronization Using Single Variable Feedback Based on Backstepping Method," Chaos Solitons and Fractals, Vol. 21, No. 5, 2004, pp. 1183-1193. http://dx.doi.org/10.1016/j.chaos.2003.12.079

[13] J. A. Lu, X. Q. Wu, X. P. Han and J. H. Lü, “Adaptive Feedback Synchronization of a Unified Chaotic System," Physics Letters A, Vol. 329, 2004, pp. 327-333. http://dx.doi.org/10.1016/j.physleta.2004.07.024

[14] F. X. Chen, C. S. Zhang, G. J. Ji, S. Zhai and S. Zhou, "Chua System Chaos Synchronization Using Single Variable Feedback Based on Lasalle Invariance Principal," Proceedings of the 2010 IEEE International Conference on Information and Automation, Harbin, 20-23 June 2010, pp. 301-304.

[15] P. Bergé, Y. Pomeau and C. Vidal, "Order within Chaos: Towards a Deterministic Approach to Turbulence,” John Wiley \& Sons, New York, 1984.

[16] H. K. Khalil, "Nonlinear Systems," Third Edition, Publish House of Electronics Industry, Beijing, 2007. 